\title{
Adaptation of Transcatheter Aortic Valve Replacement in Nursing
}

\author{
Nami B. Osterwald, Raymund Gantioque \\ Patricia A. Chin School of Nursing, California State University, Los Angeles, USA \\ Email: namiosterwald@gmail.com
}

How to cite this paper: Osterwald, N.B. and Gantioquez, R. (2020) Adaptation of Transcatheter Aortic Valve Replacement in Nursing. Open Journal of Nursing, 10, 396-410.

https://doi.org/10.4236/ojn.2020.104027

Received: February 28, 2020

Accepted: April 24, 2020

Published: April 27, 2020

Copyright $\odot 2020$ by author(s) and Scientific Research Publishing Inc. This work is licensed under the Creative Commons Attribution International License (CC BY 4.0).

http://creativecommons.org/licenses/by/4.0/

(c) (i) Open Access

\begin{abstract}
Transcatheter aortic valve replacement (TAVR), which is also referred to as transcatheter aortic valve implantation (TAVI), is a minimally invasive procedure to treat aortic stenosis. An estimated $12 \%$ of individuals over the age of 75 have aortic stenosis, and it is currently the most common reason for valve replacement in elderly Americans. TAVR was introduced as an alternative treatment to surgical aortic valve replacement (SAVR), which involves an open-heart surgery. In the U.S., the first transcatheter valve was approved by the U.S. Food and Drug Administration (FDA) in November 2011. Since then, FDA has expanded its approval to intermediate- and low-risk patients. The purpose of this manuscript is to expand nurses' knowledge about the TAVR procedure as TAVR is one of the most rapidly growing medical procedures in the U.S. This manuscript provides a background of the TAVR procedure, reviews relevant research, and highlights common complications. Compared to SAVR, TAVR has established its safety, efficacy, and cost-effectiveness as a treatment option for patients with severe aortic stenosis.
\end{abstract}

\section{Keywords}

Transcatheter Aortic Valve Replacement, TAVR, Transcatheter Aortic Valve Implantation, TAVI, Aortic Stenosis

\section{Introduction}

Transcatheter aortic valve replacement (TAVR), which is also referred to as transcatheter aortic valve implantation (TAVI), is a minimally invasive procedure to treat aortic stenosis. Aortic stenosis is a valvular heart disease involving the aortic valve which regulates blood flow from the left ventricle (LV) to the aorta and subsequently to the rest of the body. Aortic stenosis causes narrowing 
of the aortic valve opening. Typical symptoms are shortness of breath, fatigue, dizziness or syncope, and chest pain [1] [2]. It is the most common valvular disease in the elderly, and if left without treatment, symptomatic severe aortic stenosis is associated with high mortality [3] [4]. An estimated $12 \%$ of individuals over the age of 75 have aortic stenosis [5] [6], and it is currently the most common reason for valve replacement in elderly Americans [3].

For decades, surgical aortic valve replacement (SAVR) was the mainstay and the only effective treatment for severe aortic stenosis [1] [3] [4] [7] [8] [9]. SAVR requires open-heart surgery with a sternotomy, cross-clamping of the aorta, and cardiopulmonary bypass [7]. The high operative mortality of SAVR kept some patients from receiving the treatment. For one third of patients with aortic stenosis who are not eligible for SAVR, medical management can improve symptoms but cannot extend life or slow disease progression [7]. Without valve replacement surgery, life expectancy is two to three years, and only $50 \%$ live more than two years after the onset of symptoms [2] [7].

TAVR was introduced as a minimally invasive procedure as an alternative treatment to SAVR for patients with severe aortic stenosis that are inoperable or have high surgical risk. The first in-human TAVR was performed in April 2002 in France [1] [2] [6] [10]. TAVR involves placing a collapsible bioprosthetic valve that is made with bovine or porcine pericardium, directly over the damaged native valve [1] [7]. The bioprosthetic valve can be deployed percutaneously or through a small incision in the chest wall [7]. The replacement valve is fed over a guidewire to the aorta and wedged into the valve site. The procedure normally takes four to five hours and is done in a hybrid cardiac catheterization laboratory [7]. A patient's experience with TAVR may be somewhat similar to a stent placement in an artery in terms of recovery, which requires a shorter hospital stay [11]. As described, the differences between SAVR and TAVR are significant.

With advances in medical treatments and aging of the population, there are more elderly patients living with severe aortic stenosis and other comorbidities. Currently, in the U.S., the number of patients undergoing TAVR exceeds SAVR [3]. As the population eligible for TAVR expands, nurses in various fields are more likely to encounter patients who underwent or will undergo TAVR. Normally, post-procedural TAVR patients are monitored at an intensive care unit (ICU). As TAVR is becoming the standard treatment, hospitals are developing and implementing more efficient and cost effective fast-track TAVR protocols without compromising patient outcomes. For example, with a fast-track post-TAVR care protocol, patients stay in a post-anesthesia recovery unit for two to four hours after the TAVR procedure [1]. They are then transferred to a telemetry unit rather than to the ICU, and discharged the next day. These new trends with fast-track TAVR protocols require nurses to understand the TAVR procedure and its post-procedural care to ensure the best patient outcomes.

The purpose of this manuscript is to help nurses understand the TAVR pro- 
cedure and prepare them to provide care for the patients who have undergone TAVR, whether post-procedurally or in ongoing care. Through a review of evidence-based literature, the background of TAVR, relevant research, and associated complications are explored to expand nurses' knowledge and maximize patient outcomes.

\section{Background of TAVR}

In the U.S., TAVR began in May 2007 with a randomized trial for TAVR vs. SAVR [1]. In the trial, patients with very high surgical risk were randomized to either TAVR or SAVR [4]. Based on clinical trials, TAVR demonstrated comparable or even superior results compared with SAVR [3] [12].

In November 2011, the first transcatheter valve (balloon-expandable) received approval from the U.S. Food and Drug Administration (FDA) for surgically inoperable patients [1] [13] [14]. In the U.S., the Society of Thoracic Surgeons (STS) Predicted Risk of Mortality (PROM) score is utilized to assess risk of death during the first 30 days after cardiac surgery based on baseline patient characteristics [2] [7] [14]. An STS-PROM score of $8 \%$ or greater is considered high risk for surgical mortality [1] [2] [14] [15], and such patients were candidates for a TAVR procedure.

Another milestone for TAVR was in March 2015 when the FDA approved placing a TAVR valve inside a bioprosthetic valve (valve-in-valve) [7] [14]. This expanded the treatment options for many patients. Before valve-in-valve TAVR was approved, younger patients were considered to be better candidates for a mechanical valve (i.e., SAVR) since their life expectancy was longer than the life expectancy of a bioprosthetic valve [1]. Valve-in-valve TAVR offers an option for these patients knowing that placement of a TAVR valve inside a failing bioprosthetic valve will be possible rather than redoing an open-heart surgery at an older age with higher risk [1].

In August 2016, the FDA approved one of the valves for intermediate-risk patients [1] [14]. Intermediate risk is defined as an STS-PROM score of $4 \%$ to $8 \%$ [14] [15]. In August 2019, the FDA expanded its approval for some TAVR valves for low risk patients with their STS-PROM score of less than 4\% [16]. When TAVR was approved in the U.S. in 2011, the requirements for hospitals developing a TAVR program were strict and facilities were required to adhere to Medicare guidelines. A recent study reported that more than 25,000 TAVRs are being performed annually across more than 400 centers in the U.S. [5]. Compared with SAVR, TAVR has reportedly led to decreased length of stay (LOS), reduced risks, and reduced costs without affecting outcomes [1] [17]. Currently, there are four different commercial TAVR valves from two companies available, and several other transcatheter valves are in different phases of development [1] [7]. Additionally, TAVR has been approved and eligible for high-risk and intermediate-risk patients receiving Medicare and Medicaid [11] [14].

TAVR was initially considered to have limited cost-effectiveness [8] [12]. The cost of a TAVR prosthesis valve is approximately $\$ 32,000$ while the cost of a 
SAVR valve is approximately $\$ 5000$ [8] [18]. After in-hospital costs were added to the procedural costs, the total hospitalization costs between TAVR and SAVR were comparable and approximately $\$ 55,000$ [6] [18] [19]. This was because the costs for the hospitalization were substantially lower with TAVR than with SAVR mainly because of a reduction in LOS [8] [18] [19]. When 1-year cumulative costs were considered, the average cost of TAVR was $\$ 15,000$ less than the average cost of SAVR ( $\$ 80,977$ vs. $\$ 96,489)$ [18]. In this cost-effectiveness analysis, TAVR has shown to be cost-effective in the high-risk population and cost-saving in the intermediate-risk population [8]. The differences between TAVR and SAVR are highlighted in Table 1.

\section{TAVR Procedure}

The first step of a TAVR procedure involves identifying a patient's overall surgical risk with the STS-PROM score [7]. Once the TAVR team evaluates the patient's surgical risk, a decision is made for whether SAVR or TAVR is the patient's best option [7]. If TAVR is selected, a pre-TAVR workup is completed. A pre-TAVR workup includes, but not limited to:

Table 1. TAVR vs. SAVR.

\begin{tabular}{|c|c|c|}
\hline & TAVR & SAVR \\
\hline Inception & 2011 (in the U.S.) & 1960 \\
\hline $\begin{array}{l}\text { Eligible Population } \\
\text { (STS-PROM Score) }\end{array}$ & $\begin{array}{l}\text { High risk }(>8 \%) \text {, intermediate } \\
\text { risk }(4 \%-8 \%) \text {, low risk }(<4 \%)\end{array}$ & $\begin{array}{l}\text { Intermediate risk }(4 \%-8 \%) \text {, low } \\
\text { risk }(<4 \%) \text {, younger patients } \\
{ }^{*} 1 / 3 \text { of patients not eligible }\end{array}$ \\
\hline \multirow[t]{4}{*}{ Procedure } & Minimally invasive procedure & $\begin{array}{l}\text { Requires full sternotomy or less } \\
\text { invasive mini-sternotomy, } \\
\text { cardiopulmonary bypass, } \\
\text { cross-clamping of the aorta }\end{array}$ \\
\hline & $\begin{array}{l}\text { Bioprosthetic valve is placed } \\
\text { directly over the damaged } \\
\text { native valve }\end{array}$ & Native stenotic valve is removed \\
\hline & $\begin{array}{l}\text { Small vascular punctures } \\
\text { (transfemoral) }\end{array}$ & Large surgical incision \\
\hline & $\begin{array}{l}\text { Conscious sedation with local } \\
\text { anesthesia (transfemoral) }\end{array}$ & $\begin{array}{l}\text { General anesthesia with } \\
\text { intubation }\end{array}$ \\
\hline Procedural Setting & Cardiac catheterization lab & Operating room \\
\hline Average Procedure Duration & 84 mins & $236 \mathrm{mins}$ \\
\hline Average Length of Stay & 4.6 days & 10.9 days \\
\hline Valve Cost & $\$ 32,000$ & $\$ 5,000$ \\
\hline $\begin{array}{l}\text { Average Total Admission } \\
\text { Costs (including procedure) }\end{array}$ & $\$ 54,256$ & $\$ 58,410$ \\
\hline $\begin{array}{l}\text { Average 1-Year Cumulative } \\
\text { Costs }\end{array}$ & $\$ 80,977$ & $\$ 96,489$ \\
\hline 2-Year Mortality & $13.1 \%$ & $17.1 \%$ \\
\hline
\end{tabular}


- CT angiogram of the chest, abdomen, and pelvis;

- transthoracic echocardiogram (TTE) or transesophageal echocardiogram (TEE);

- right and left cardiac catheterization;

- carotid duplex ultrasonography;

- pulmonary functions tests (PFTs) if the patient has severe chronic obstructive pulmonary disease (COPD);

- standard laboratory work;

- frailty assessment; and

- Kansas City Cardiomyopathy Questionnaire [1] [2].

There are various approved TAVR approaches; however, about $90 \%$ of TAVR procedures utilize a minimally invasive percutaneous transfemoral approach [7]. Most transfemoral TAVR cases use conscious sedation [1]. The transfemoral approach may not be appropriate for patients with peripheral vascular disease due to potential issues with their vessel sizes [7]. The other percutaneous approaches are transaxillary or subclavian [7]. Traditional open approaches are also available which require small surgical incisions (i.e., minithoracotomy) and intubation [7]. Open approaches include transapical and transaortic [7].

Overall, $98 \%$ to $99 \%$ of TAVR procedures go smoothly without peri-procedural complications [1]. Moreover, as TAVR centers have gained more experience, some facilities have begun to simplify and optimize their TAVR programs in order to respond to the growing worldwide need for TAVR. A TAVR center in Copenhagen, Denmark, developed a minimalist TAVR procedure and fast-track TAVR course that has distinct advantages over the more traditional approach without compromising safety and clinical effectiveness [10].

As a critical part of fast-track TAVR course, Chopra et al. adopted local anesthesia or conscious sedation for the procedure, which is as safe as general anesthesia [10]. The use of local anesthesia or conscious sedation with TAVR became routine because the use of peri-procedural TEE to assess for a perivalvular leak (PVL) was found to be no longer necessary [10]. Based on several studies, clinical outcomes are similar for TAVR under local anesthesia without TEE compared to TAVR under general anesthesia with TEE [10].

Another strategy for the simplification of TAVR by Chopra et al. implemented "no catheters except for one peripheral venous line" [10]. According to Chopra et al., a recent observational TAVR study suggested that avoiding urinary catheterization minimizes in-hospital complications by significantly lowering rates of urinary infection during the hospital stay [10]. Additionally, the use of a central venous line also has its own complications and is best avoided when possible [10].

Early mobilization is another key to the fast-track TAVR course. Since the majority of TAVR cases without general anesthesia do not require post-procedural monitoring at an ICU, the patients can be transferred to the telemetry floor after completion of the procedure, avoiding the ICU [1] [10] [12]. This eliminates a large burden on the ICU and results in a significant cost reduction [10]. Early 
mobilization from six hours after the procedure and discharge within 24 to 48 hours reduces the risk of post-procedure delirium among patients and subsequently leads to a faster improvement in the patient's quality of life [10]. Patients who underwent alternative access (i.e., other than transfemoral) or those with peri-procedural complications should be monitored at an ICU during the post-procedural period [10].

At the facility in Copenhagen, a total of 543 patients underwent transfemoral TAVR with the minimalist approach between 2016 and 2017 [10]. In total, 314 (57.8\%) patients were discharged within 48 hours while 43 (7.9\%) patients were hospitalized for more than five days [10]. Based on the study, a short post-procedural LOS was not associated with an increased risk of readmission within 30 days or a year [10]. In comparison, the risk of one-year readmission increased with longer post-procedural LOS [10].

A fast-track or minimalist TAVR protocol has not yet been reported in the U.S. except for one small attempt by the Mid-Atlantic Permanente Medical Group. Galper et al. developed and implemented a fast-track post-TAVR care protocol in February 2017 with a focus on next-day discharge [20]. Their fast-track protocol included the removal of central lines at the end of the TAVR procedure, recovery in a non-ICU setting, and ambulation by the evening of the procedure [20]. Patients with significant LV or RV dysfunction, severe COPD or pulmonary hypertension, body mass index over 50 , and planned alternative access were excluded from the fast-track protocol [20].

In Galper's study, 35 patients were treated with their fast-track protocol with an average age of 82.0 years and an average STS-PROM score of $6.6 \%$ [20]. Of these 35 patients, 28 patients remained in the fast-track protocol through discharge with an average LOS of 1.4 days [20]. The overall LOS was reduced from 3.2 days prior to the implementation of the protocol to 1.9 days indicating that a fast-track TAVR protocol can lead to a decreased LOS with frequent next-day discharge [20].

\section{Relevant TAVR Research}

In the U.S., TAVR is a fairly new procedure and is still in its first decade since its introduction. Available studies involving TAVR tend to be limited to retrospective statistical analyses targeted for interventional cardiologists. Many of these studies compare the different types of bioprosthetic valves and procedural techniques. Although this kind of information has a limited impact on nursing scope of practice, some recent studies offer TAVR data that help nurses further understand the TAVR procedure, as TAVR is one of the most rapidly growing medical procedures in the U.S.

\subsection{Trends in SAVR vs. TAVR Outcomes}

Kundi et al. conducted a retrospective statistical study of Medicare beneficiaries who underwent aortic valve replacement, either SAVR or TAVR, between January 1, 2009, and December 31, 2014 [3]. This study period includes the pre-TAVR 
period (i.e., only SAVR was available), and patients aged 65 and older were included [3]. Out of 137,563 hospitalizations that were isolated for aortic valve replacement during the study period, 102,968 (74.9\%) were for SAVR and 34,595 (25.1\%) were for TAVR [3].

During the study period, the volume of both SAVR and TAVR increased after the introduction of TAVR in 2011 [3]. TAVR volumes increased in all age groups with the largest increase in those older than 85 years old ( 0.5 per 100,000 beneficiaries in 2011 to 13.8 in 2014) [3]. The mean age of aortic valve replacement recipients increased significantly after the introduction of TAVR from 76.6 years in 2009 to 79.0 years in 2014 [3].

Over the study period, predicted 30-day mortality in overall patients increased from $4.0 \%$ in 2009 to $5.4 \%$ in 2014 , with the largest increase in the age group over 85 years (5.9\% in 2009 to $6.6 \%$ in 2014) [3]. Among SAVR patients, predicted 30 -day mortality decreased ( $4.0 \%$ in 2009 to $2.9 \%$ in 2014 ) while predicted 30-day mortality among TAVR patients remained stable $(6.7 \%$ in 2009 assuming treatment with SAVR, to $6.3 \%$ in 2014) [3]. These results suggest that high-risk patients shifted from SAVR to TAVR reduced the predicted 30-day mortality among SAVR patients while overall 30-day mortality increased slightly due to broadened eligibility for aortic valve replacement in high-risk patients after the introduction of TAVR [3].

\subsection{Impact on Readmission to Index Hospitals vs. Non-Index Hospitals}

Since TAVR recipients are older and tend to have multiple comorbidities, there is a relatively high 30 -day readmission rate of $15 \%$ to $18 \%$ [21]. Driven by a negative impact on clinical and financial outcomes, Ando et al. conducted a statistical study to clarify this issue. Since TAVR is often performed at large hospitals, readmission to index hospitals (i.e., where the initial procedure was performed) could be limited to the TAVR population due to difficult geographical access and socioeconomic factors [21]. This study compared the impact of clinical and financial outcomes on 30-day non-elective readmissions after TAVR between index and non-index hospitals.

Data from the Nationwide Readmission Database was collected from 22 individual inpatient databases, which represent approximately 50\% of nationwide hospitalizations [21]. From January 2012 to September 2015, a total of 44,929 patients who were age 50 or older, survived after TAVR and were discharged, were included in the study [21]. Of these patients, 6808 (14.5\%) had non-elective 30-day readmissions [21]. Of this total, 4244 (62.3\%) were admitted to the index hospitals, while 2564 (37.7\%) were admitted to non-index hospitals [21].

As expected, patients that reside in counties with a smaller population were more likely to be readmitted to non-index hospitals [21]. However, the study shows there was no difference in in-hospital mortality between index and non-index readmission [21]. The rates for other readmission outcomes, such as acute myocardial infarction, pacemaker placement, stroke, and acute kidney in- 
jury, were similar in both index and non-index hospitals except for bleeding requiring transfusion, which was higher in non-index readmissions $(23.8 \%$ vs. 17.1\%) [21]. LOS for readmission was also comparable between index hospitals (5.65 days) and non-index hospitals (5.70 days) as well as cost of readmissions ( $\$ 15,410$ vs. $\$ 16,390$ respectively) [21]. The most common reason for readmission in both groups was congestive heart failure [21].

This study indicates that a large portion of the TAVR population (37.7\%) does not have access or resources to present to the index hospital when readmission is inevitable. These patients tend to wait longer before being readmitted [21]. Nonetheless, the outcomes are similar and there is not a significant negative impact when patients are readmitted to non-index hospitals. These results are reassuring for patients with limited options for readmission after TAVR.

\subsection{TAVR Volume and Patient Outcomes}

Statistical research conducted by Vemulapalli et al. investigated the relationship between the hospital volume of TAVR procedures and patient outcomes. This was a follow up study to evaluate whether the associated relationship persists after the patient population was expanded for TAVR and the technology and techniques were improved since previous research. The authors also examined the effect of hospitals' "start-up" period and whether patient characteristics or hospital characteristics vary based on the hospital's procedural volume [13].

This study used data from the STS-American College of Cardiology (ACC) Transcatheter Valve Therapy (TVT) Registry for all TAVR procedures that were performed between January 1, 2015 and December 31, 2017. Hospitals are required by the Centers for Medicare and Medicaid Services to submit patient data to the TVT Registry for those who received a commercially approved TAVR device [13]. The study included 92,256 transfemoral TAVR procedures at 554 sites by 2935 operators [13].

This study concluded that there was a significant nonlinear association between hospital procedural volume in transfemoral TAVR and mortality [13]. However, there was no association between hospital procedural volume and the 30-day complications, except for major vascular complications or major bleeding [13]. The relationship between procedural volume and vascular complications or major bleeding is also seen in percutaneous coronary intervention despite technology improvements [13]. This relationship between lack of hospital experience and bleeding complications is also consistent with the index vs. non-index readmissions study by Ando et al. (i.e., hospitals less experienced with TAVR have a higher risk of bleeding complications). Based on the study results, hospital procedural volume can be a factor to consider when a patient decides to proceed with TAVR procedure and has options for available hospitals.

In addition to the persistent association between procedural volume and 30-day mortality, this study further revealed that the association remains even after excluding the 6-month and 12-month "start-up" periods at each hospital [13]. Hospitals with a lower procedural volume were more likely located in rural 
areas, and these hospitals treated a greater proportion of the Black and Hispanic patient population [13]. Compared to the previous study, this study reported a major improvement in 30-day mortality in transfemoral TAVR, which decreased from $7.5 \%$ in 2012 to $2.5 \%$ to $3.0 \%$ in their study period [13].

\subsection{TAVR in Low-Risk Patients}

In another recent study, Kolte et al. looked into TAVR in low-risk patients. TAVR has established its safety and efficacy as a treatment option for patients with severe aortic stenosis with high or intermediate risk for SAVR. Meanwhile, SAVR remains the standard therapy in current clinical practice for low-risk patients and younger patients [5]. There are conflicting findings in observational studies comparing TAVR and SAVR in low-risk patients [5].

Kolte et al. conducted a meta-analysis with three randomized controlled trials (RCT) and one post-hoc analysis of an RCT, which included a total of 2887 low-risk patients from the inception of the case (with unspecified date) through March 20, 2019 [5]. The low-risk patients are defined as having an STS-PROM score of less than 4\% [5] [14]. 1497 patients were randomized to TAVR and 1390 were randomized to SAVR among 4 RCT studies [5]. The mean age of these patients was 75.4 years, and the mean STS-PROM score was 2.3\% [5].

The primary focus of this study was on the one-year outcomes and found that TAVR was associated with a significantly decreased risk of death from any cause compared with SAVR (2.1\% vs. 3.5\%) [5]. Rates of stroke were similar in both groups, and TAVR was associated with higher rates of permanent pacemaker implantation and moderate to severe PVL at one year compared to SAVR [5]. Although this study suggests the preference of TAVR to SAVR for low-risk patients, the researchers are cautious about the use of their study to justify TAVR in young patients. Most of the study participants were elderly and this meta-analysis did not use individual-level data. It is also important to note that long-term valve durability is still uncertain [5] [16].

\section{TAVR Complications}

As TAVR expands rapidly, more nurses are expected to encounter post-procedural patients or patients that previously underwent TAVR. TAVR appears to be a miracle cure to those who otherwise did not have a treatment option, but there are risks accompanying the procedure. Some studies report that up to one third of patients experience complications after TAVR [7]. Data from a newer valve (SAPIEN 3) registry reported 30-day mortality was $1.1 \%$ [22]. Understanding the common complications that are associated with TAVR is important when providing care to these patients.

\subsection{Peri-Procedural Complications}

A German study, which focused on the peri-procedural complications, concluded that TAVR is safe and with acceptable complication rates [23]. Based on the study, $1 \%$ of the 15,964 patients that were included in the research data died 
during the procedure or on the day of the procedure [23]. The patients who died peri-procedurally were significantly different from all other patients [23]. 5\% of the patients experienced severe vital complications other than death on the day of the procedure, including conversion to sternotomy, acute percutaneous coronary intervention, low cardiac output requiring mechanical circulatory support, cardiac tamponade requiring treatment, aortic dissection, or annular rupture [23]. The overall survival rate for severe vital complications was 59.2\% [23].

\subsection{Cerebrovascular Accidents (CVAs) after TAVR}

Although the CVA/transient ischemic attack (TIA) rate in patients with TAVR was approximately double compared to that with SAVR from the original trial, recent data showed similar rates of disabling strokes within two years after the procedures [1]. Based on the study by Mastoris et al., which focused on CVAs after TAVR, acute and subacute CVAs after TAVR were reported in $3 \%$ to $6 \%$ of patients [4]. Mastoris et al. reviewed multiple studies and confirmed that CVAs after TAVR peak in the immediate post-procedure period, with a steady decline over the following months [4]. According to a meta-analysis of RCTs, the early CVA rate (less than 30 days) was as low as $2.9 \%$ [4].

Among the CVAs after TAVR, $45 \%$ of CVAs occur within two days after the procedure, 28\% between 3 and 10 days, $4 \%$ between 10 and 30 days, and 10.5\% from one month to two years [4]. As hospitalization after the procedure has been shortened and the next day discharge is becoming the new common goal for TAVR programs, nurses and other caregivers have a crucial role in monitoring patients and recognizing the early signs of CVAs after TAVR.

Antithrombotic treatment is recommended for the prevention of ischemic CVAs during and after TAVR [2] [4] [7] [10]. One RCT study recommends 75 to $100 \mathrm{mg}$ of daily aspirin, a $300 \mathrm{mg}$ of clopidogrel loading dose, and $75 \mathrm{mg}$ daily dose for six months following TAVR [4]. However, the loading dose and duration of clopidogrel as well as efficacy of dual antiplatelet therapy (DAPT) have not been well defined under current guidelines [4] [6]. In fact, some studies reported that DAPT did not reduce the incidence of new CVAs, but it was associated with a significantly higher rate of major and life-threatening bleeding complications [4] [6].

\subsection{Vascular Complications}

The most common vascular access complication is hematoma [7]. Although vascular injuries are less common now than in the early days of TAVR, there remains a $1 \%$ to $2 \%$ chance of damage to an iliac or femoral artery during a transfemoral approach [1]. Usually, a small incision is made in bilateral femoral regions. One of the incisions is used to introduce the prosthetic valve and the other used for placement of a temporary transvenous pacemaker [2]. If the patient does not experience new onset of atrioventricular (AV) block during the procedure, the pacemaker is removed at the end of the procedure [2].

Hematomas develop when blood leaks from the vascular puncture point into 
the soft tissue [7]. The affected area may feel firm to palpation, appear swollen and/or discolored, and the patient may complain of tenderness or pain [7]. When hematoma is suspected, pressure should be applied 1 to $2 \mathrm{~cm}$ proximal to the puncture site because the internal arterial puncture site is proximal to the external incision [7]. The initial area of hematoma should be marked for subsequent assessments and the provider needs to be notified [7]. Subsequent assessments of the site should look for thigh enlargement, discoloration outside the marked boundaries, change in vital signs, and pain level or location [7].

Retroperitoneal bleeding is more likely to occur when the femoral artery is punctured above the inguinal ligament, commonly known as a "high-stick" [7]. Unlike hematoma, there may not be obvious signs of bleeding [7]. The clinical findings of retroperitoneal bleeding could present as back, flank, or abdominal pain, decrease in blood pressure, increased heart rate, and hemoglobin and hematocrit levels may decrease [7]. When retroperitoneal bleeding is suspected, the patient must remain on bedrest and the provider should be notified [7]. The provider may order a CT scan to diagnose the problem and a surgical repair may be necessary [7]. The administration of IV fluids or blood transfusion should be expected [7].

Arterial occlusion is another vascular complication of TAVR. Arterial occlusion has to be suspected if the patient complains of pain and/or paresthesia, exhibits pallor, has an absent pulse in the affected extremity or has the inability to move the limb [7]. The treatment of arterial occlusion depends on the size and location of the occlusion and severity of the symptoms [7]. The occlusive thrombus may lyse spontaneously, but if it does not, thrombolytic agents or a surgery may be an option if not contraindicated [7].

Major bleeding 30 or more days after TAVR is also common [22]. Based on an RCT study, 5.9\% with median onset at 132 days were reported as major bleeding being a strong independent risk factor for mortality between 30 days and one year after TAVR [22]. The most frequent types of late major bleeding were gastrointestinal (40.8\%), neurological (15.5\%), and traumatic fall-related (7.8\%) [22].

To minimize these vascular complications, frequent neurovascular monitoring and early recognition of the signs are important as well as providing effective patient education. With the shortened LOS, post-procedural TAVR patients are discharged from hospitals while they are still at high risk for these complications.

\subsection{Other Complications}

Additional complications from TAVR include high-degree AV block requiring permanent pacemaker implantation, paravalvular regurgitation (PVR), and hypotension. It was also associated with a higher incidence of permanent pacemaker implantation compared with SAVR [2] [6]. Risk factors for new onset AV block are a history of right bundle branch block as well as AV nodal delay, advanced age, low implant positioning, or an oversized prosthetic valve [2].

PVR can be caused by a mismatch of the prosthetic valve and the native valve annulus, incomplete valve positioning or sealing [7]. Newer-generation prosthetic valves have fewer PVR complications [7]. Moderate or severe PVR fol- 
lowing TAVR is associated with a threefold increase in 30-day mortality and a 2.3-fold increase in one-year mortality following TAVR [22]. Diagnosis of PVR is challenging, and TTE is mainly used [22].

Hypotension can also occur postoperatively due to volume depletion, conduction disturbances, and dysrhythmias [2]. Ideally, the patient's systolic blood pressure should be monitored and maintained at or above $100 \mathrm{mmHg}$ after the procedure [2]. Hypotension can be managed with IV fluids, low-dose inotropes, and vasopressors if needed [2].

TIA, myocardial infarction, acute kidney injury (AKI) are also common complications of TAVR [7]. However, these complications are more common with SAVR. Meanwhile for TAVR, there is a significantly lower rate of AKI, severe bleeding, and new onset of atrial fibrillation [2] [7]. Long-term complications following TAVR include PVR, endocarditis, prosthetic valve thrombosis, and bleeding [2] [22]. Common TAVR complications are summarized in Table 2.

Table 2. Summary of common TAVR complications.

\begin{tabular}{|c|c|}
\hline \multicolumn{2}{|c|}{ Periprocedural Complications: During the procedure or on the day of the procedure } \\
\hline Death & $1 \%$ \\
\hline $\begin{array}{l}\text { Severe vital complications other than death } \\
\text { (conversion to sternotomy, acute PCI, low cardiac output }\end{array}$ & $5 \%$ \\
\hline $\begin{array}{l}\text { requiring mechanical circulatory support, cardiac tamponade, } \\
\text { aortic dissection, or annular rupture) }\end{array}$ & (Survival rate $59.2 \%$ ) \\
\hline \multicolumn{2}{|l|}{ Postprocedural Complications } \\
\hline 30-day mortality & $1.1 \%-3.0 \%$ \\
\hline Cerebrovascular accident (CVA) & $3 \%-6 \%$ \\
\hline Within 2 days & $45 \%$ \\
\hline 3 - 10 days & $28 \%$ \\
\hline 10 - 30 days & $4 \%$ \\
\hline 1 month - 2 years & $10.5 \%$ \\
\hline \multicolumn{2}{|l|}{ Vascular Complications } \\
\hline \multicolumn{2}{|l|}{ Hematoma } \\
\hline \multicolumn{2}{|l|}{ Retroperitoneal bleeding } \\
\hline Arterial occlusion & \\
\hline Major bleeding 30 or more days after the procedure & \\
\hline
\end{tabular}

High-degree AV block requiring permanent pacemaker implantation Higher incidence than SAVR

Paravalvular regurgitation (PVR)

Hypotension

Transient ischemic attack (TIA)

Myocardial infarction (MI)

Acute kidney injury (AKI)

Long-Term Complications

Paravalvular regurgitation (PVR)

Endocarditis

Prosthetic valve thrombosis

Bleeding

$5.9 \%$ with median onset at 132 days 


\section{Conclusion}

The expanding eligibility and availability of TAVR affect patients with severe aortic stenosis and nursing practice. Recently, TAVR has expanded to lower-risk patients, which will lead to a larger number of patients undergoing the procedure. More hospitals are developing more efficient TAVR programs to accommodate the expanding need. While the safety and efficacy of TAVR have been proven, the risks accompanying the procedure are inevitable. Nurses have an important role in the care of TAVR patients and having knowledge about the procedure is vital for providing appropriate care. Since TAVR is a fairly new procedure in the U.S. with limited information for nursing care, it is essential that nurses stay up to date with the most current information in order to maximize patient outcomes.

\section{Conflicts of Interest}

The authors declare no conflicts of interest regarding the publication of this paper.

\section{References}

[1] Berger, D. (2018) Evolution of a TAVR Program. Critical Care Nursing Quarterly, 41, 360-368. https://doi.org/10.1097/CNQ.0000000000000221

[2] Karycki, M.K. (2019) Transcatheter Aortic Valve Replacement. Nursing, 49, 31-32. https://doi.org/10.1097/01.NURSE.0000558086.92851.51

[3] Kundi, H., Cohen, D.J., Strom, J.B., Valsdottir, L., Shen, C., Yeh, R.W., et al. (2019) Trends in Isolated Aortic Valve Replacement in the United States in the Early Phase of Expansion of TAVR. International Journal of Cardiology, 292, 68-72. https://doi.org/10.1016/j.ijcard.2019.06.061

[4] Mastoris, I., Schoos, M.M., Dangas, G.D. and Mehran, R. (2014) Stroke after Transcatheter Aortic Valve Replacement: Incidence, Risk Factors, Prognosis, and Preventive Strategies. Clinical Cardiology, 37, 756-764. https://doi.org/10.1002/clc.22328

[5] Kolte, D., Vlahakes, G.J., Palacios, I.F., Sakhuja, R., Passeri, J.J., Elmariah, S., et al. (2019) Transcatheter versus Surgical Aortic Valve Replacement in Low-Risk Patients. Journal of the American College of Cardiology, 74, 1532-1540. https://doi.org/10.1016/j.jacc.2019.06.076

[6] Tsigkas, G., Despotopoulos, S., Makris, A., Koniari, I., Armylagos, S. and Hahalis, G. (2018) Transcatheter versus Surgical Aortic Valve Replacement in Severe, Symptomatic Aortic Stenosis. Journal of Geriatric Cardiology, 15, 76-85.

[7] Haight, K. (2017) Caring for Patients after Transcatheter Aortic Valve Replacement: Understanding Options for Patients Helps You Be a More Effective Caregiver. American Nurse Today, 12, 10-15.

[8] Baron, S., Reynolds, M.R. and Cohen, D.J. (2019) Economic Considerations for TAVR vs. SAVR: Historical Perspective and Future Predictions. American College of Cardiology. https://www.acc.org/latest-in-cardiology/articles/2019/06/18/07/43/economic-consi derations-for-tavr-vs-savr

[9] Ramlawi, B., Ramchandani, M. and Reardon, M.J. (2014) Surgical Approaches to 
Aortic Valve Replacement and Repair: Insights and Challenges. Interventional Cardiology Review, 9, 32-36. https://doi.org/10.15420/icr.2011.9.1.32

[10] Chopra. M., Luk, N.H.V., Backer, O.D. and Søndergaard, L. (2018) Simplification and Optimization of Transcatheter Aortic Valve Implantation-Fast-Track Course without Compromising Safety and Efficacy. BMC Cardiovascular Disorders, 18, 1-9. https://doi.org/10.1186/s12872-018-0976-0

[11] American Heart Association (2019) What Is TAVR? https://www.heart.org/en/health-topics/heart-valve-problems-and-disease/understa nding-your-heart-valve-treatment-options/what-is-tavr

[12] Marcantuono, R., Gutsche, J., Burke-Julien, M., Anwaruddin, S., Augoustides, J.G., Herrmann, H.C., et al. (2015) Rationale, Development, Implementation, and Initial Results of a Fast Track Protocol for Transfemoral Transcatheter Aortic Valve Replacement (TAVR). Catheterization and Cardiovascular Interventions, 85, 648-654. https://doi.org/10.1002/ccd.25749

[13] Vemulapalli, S., Carroll, J.D., Mack, M.J., Li, Z., Dai, D., Bavaria, J.E., et al. (2019) Procedural Volume and Outcomes for Transcatheter Aortic-Valve Replacement. The New England Journal of Medicine, 380, 2541-2550. https://doi.org/10.1056/NEJMsa1901109

[14] Centers for Medicare \& Medicaid Services (2019) Decision Memo for Transcatheter Aortic Valve Replacement (TAVR) (CAG-00430R).

https://www.cms.gov/medicare-coverage-database/details/nca-decision-memo.aspx ?NCAId $=293 \& \mathrm{bc}=$ ACAAAAAAQAAA\&

[15] Balan, P., Zhao, Y., Johnson, S., Arain, S., Dhoble, A., Nguyen, T., et al. (2017) The Society of Thoracic Surgery Risk Score as a Predictor of 30-Day Mortality in Transcatheter vs Surgical Aortic Valve Replacement: A Single-Center Experience and Its Implications for the Development of a TAVR Risk-Prediction Model. Journal of Invasive Cardiology, 29, 109-114.

[16] U.S. Food \& Drug Administration (2019) FDA Expands Indication for Several Transcatheter Heart Valves to Patients at Low Risk for Death or Major Complications Associated with Open-Heart Surgery.

https://www.fda.gov/news-events/press-announcements/fda-expands-indication-se veral-transcatheter-heart-valves-patients-low-risk-death-or-major

[17] Arora, S., Strassle, P.D., Kolte, D., Ramm, C.J., Falk, K., Vavalle, J.P., et al. (2018) Length of Stay and Discharge Disposition after Transcatheter versus Surgical Aortic Valve Replacement in the United States. Circulation: Cardiovascular Interventions, 11, e006929. https://doi.org/10.1161/CIRCINTERVENTIONS.118.006929

[18] Baron, S., Wang, K., House, J., Magnuson, E.A., Reynolds, M.R., Cohen, D.J., et al. (2019) Cost-Effectiveness of Transcatheter versus Surgical Aortic Valve Replacement in Patients with Severe Aortic Stenosis at Intermediate Risk: Results from the PARTNER 2 Trial. Circulation, 139, 877-888. https://doi.org/10.1161/CIRCULATIONAHA.118.035236

[19] Meduri, C., Chung, J., Gaffney, J., Henley, S., Williams, J. and Gada, H. (2017) Comparison of U.S. Hospital Costs Between Transcatheter Aortic Valve Replacement (TAVR) and Surgical Aortic Valve Replacement (SAVR). Journal of the American College of Cardiology, 70, B268. https://doi.org/10.1016/j.jacc.2017.09.670

[20] Galper, B.Z., Golden, J., Rhee, J., Garrett, J. and Kulkarni, A. (2018) Implementation of a Post-Transcatheter Valve Replacement (TAVR) Fast-Track Care Protocol with a Focus on Next-Day Discharge. Journal of the American College of Cardiology, 71, A1224. https://doi.org/10.1016/S0735-1097(18)31765-0 
[21] Ando, T., Adegbala, O., Villablanca, P., Akintoye, E., Ashraf, S., Briasoulis, A., et al. (2019) Incidence and Predictors of Readmissions to Non-Index Hospitals after Transcatheter Aortic Valve Replacement and the Impact on in-Hospital Outcomes: From the Nationwide Readmission Database. International Journal of Cardiology, 292, 50-55. https://doi.org/10.1016/j.ijcard.2019.04.056

[22] Dalby, M. and Panoulas, V. (2019) Transcatheter Aortic Valve Implantation: Complications. UpToDate.

https://www.uptodate.com/contents/transcatheter-aortic-valve-implantation-compl ications

[23] Walther, T., Hamm, C. W., Schuler, G., Berkowitsch, A., Kötting, J. and Möllmann, H. (2015) Perioperative Results and Complications in 15,964 Transcatheter Aortic Valve Replacements. Journal of the American College of Cardiology, 65, 2173-2180. https://doi.org/10.1016/j.jacc.2015.03.034 